\title{
Sedimentological and Lithostratigraphic Study of the Senonian Series in Timstiguite Region, Central High Atals, Morocco
}

Abdelouhed Farah ${ }^{1 *}$, Ahmed Algouti 2 Abdellah Algouti ${ }^{3}$

${ }^{123}$ University of Cadi Ayyad, Faculty of Sciences Semlalia, Department of Geology, Geoscience Geotourism Natural Hazards and Remote Sensing Laboratory (2 GRNT), BP 2390, 40000, Marrakech, Morocco

Corresponding Author: Abdelouhed Farah, E-mail: farah6faragh@gmail.com

\section{ARTICLE INFORMATION}

Received: November 02, 2020

Accepted: January 05, 2021

Volume: 2

Issue: 1

DOI: 10.32996/jeas.2021.2.1.1

\section{KEYWORDS}

Sedimentology, Senonian series, Timstiguite Region, Central High Atlas of Morocco

\section{ABSTRACT}

In this paper, we present the sedimentological, stratigraphic and paleogeographic aspect of the Upper Cretaceous red formations (Senonian series) of Timstiguite region (Central High Atlas of Morocco). The formations were measured bed by bed and sampled in a very detailed way. An exoscopic analysis of the quartz grains was carried out by a systematic prospecting of the grains relief and theirs surface characteristic, this led us to characterize their origin and consequently to determine the different paleoenvironnements. The clay sediments of these formations seem to be a favorable prospect in point of view: evolution of the clay and non-clay sediments in this central basin of the High Atlas will depend mainly on the conjugation of tectonic, eustatic, climatic and other influences. The senonian thickness is more important than other sub basins (Albban and Imider) where it shows a very reduced thickness. The Senonian of Timstiguite corresponds to a succession of detrital and evaporitic carbonate facies, which confirms a coastal depositional environment with passages of some periods of marine emersions.

\section{Introduction}

Le Sénonien se présente au Maroc sous quatre faciès (Choubert, G., Du Dresnay, R., \& Hindermeyer, J. (1950). dans les fausse des HAHA et la région de Khouribga, c'est un ensemble de marne grise devenant jaune aux affleurements, on les retrouve aussi dans les Moyen Atlas. Le long des deux massifs centraux du Haut Atlas ainsi que dans le Maroc oriental, on rencontre un régime mixte comportant des formations continentales, et lagunaire précédant une série marine ou alternant avec des formations marines marneuses et calcaires. Généralement un régime mixte qui passe latéralement à l'Est à un régime lagunaire ou laguno-continental. Ce passage latéral s'observe sur deux flancs du Haut Atlas occidental et central. La zone de passage peut montrer encore des influences marine plus en plus importantes : ce sont tantôt des couches de marne verte, tantôt des grès jaunes. Vers l'est, elles cèdent la place à des formations lagunaires, apparaissant sur de grandes distances, au sein des dépôts continentaux.4-le long du flanc Sud du Haut Atlas oriental dans le sillon Pré africain (Ksar es Souk-Boudnib) ainsi que dans la partie SE des haut plateaux (Chott Tigri) on ne connait qu'un Sénonien rouge entièrement continental. Se sont généralement de grès plus rarement des marnes rouges. Sur le versant Nord du Haut-Atlas Occidental BRIVES avait trouvé une Ammonite: Mortoniceras (Texanites) texanum qui date le Santonien (Brives, A. (1919). Les premières études géologiques de détail de la région ont été lance par(Moret, 1925) en 1957, à la suite des recherches de la Société Chérifienne de Pétrole concernant particulièrement les séries crétacées du Maroc, DUFFAUD et RIVIER ont décrit une coupe à Imin'Tanout sur le versant Nord du Haut-Atlas Occidental. Donne une étude stratigraphique du bassin sédimentaire du Sud-Ouest Marocain. II faut attendre l'année 1963, pour voir apparaître une synthèse d'un travail d'AMBROGGI, qui a duré une quinzaine d'années, sous forme de Doctorat d'Etat. Son travail concernait la partie méridionale du Haut-Atlas Occidental et la plaine du Souss. II a pu apporter des précisions stratigraphiques sur le Sénonien grâce à des découvertes paléontologiques nouvelles fondées essentiellement sur des niveaux à Ammonites. Il a daté ainsi le Coniacien par Hemitissotia turzoi KARR et Plesiotissotia dullai KARR, le Santonien par Texanites texanum ROEM, le Maastrichtien par Baculites sp, tandis que le Campanien a été daté uniquement par la microfaune, en absence des Ammonites. Durant les années 1971 un
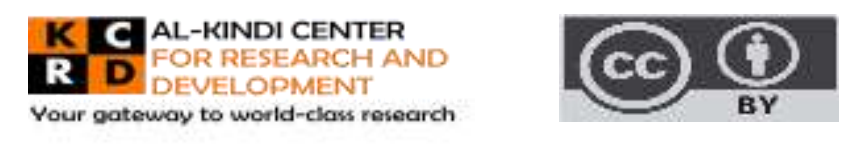

Published by Al-Kindi Center for Research and Development. Copyright (c) the author(s). This open access article is distributed under a Creative Commons Attribution (CC-BY) 4.0 license 
découpage stratigraphique a du Mésozoïque et du Cénozoïque du bassin Sud-Ouest Marocain a été proposé et draissé les premières cartes géologiques au 1/100 000 de la région (DUFFAUD, J., HEROS, R., GAUDI.1971).En 1975-1982, une équipe de l'institut géologique de l'Université de Bonn en collaboration avec WIEDMANN et son groupe (Behrens et al., 1978; Stets \& Wurster, 1982)ont proposé une reconstitution paléogéographiques du Crétacé, tout en résumant l'histoire structurale du Golfe Atlantique et sa relation avec l'ouverture de l'Océan Atlantique, depuis le Trias jusqu'au Cénozoïque, et en donnant une subdivision stratigraphique des séries crétacées. (FERRANDINI, J., \& LEMARREC, A. (1982) ont montré l'allochtonie de la couverture mésozoïque (Jurassique à Paléogène) de certaines cuvettes d'Aït Ourir (versant Nord du Haut-Atlas de Marrakech). a mis en évidence l'existence, au Sénonien, d'une sebkha littorale fortement subsidente à l'Est de la cuvette de Jbel Sour dans la région d'Ait ourir (versant Nord du Haut Atlas de Marrakech) (AZZAOUI, 1986). En 1988, OUGARANE a étudié deux coupes du Sénonien du versant Sud du HautAtlas Occidental, dans la région d'Imini (Gara Assaoud et Ste Barbe). II en a dégagé un milieu "lagunaire", entrecoupé par deux épisodes marins à sédimentation oobioclastique. Les faciès rencontrés montrent une sédimentation détritique (grès et microconglomérat) formé dans un milieu fluviatile et une autre évaporitique reçoivant des décharges oobioclastiques dans un milieu lagunaire. En 1989, IÇAME après son étude biostratigraphique de la coupe d'IminTanout étalée depuis l'Albien jusqu'au Maestrichtien, a remarqué une lacune probable, du Santonien et du Campanien, avec un dépôt maastrichtien reposant directement sur le Coniacien. (Daoudi, 1991), en étudiant les assemblages argileux des sédiments du Jurassique supérieur à l'Eocène, leurs modifications diagénétiques et leurs influences vis-à-vis de l'évolution des environnement de dépôt dans le Golfe atlantique, a montré que le Crétacé supérieur correspond à une période stable qui permet le développement de couvertures pédologiques à smectite et l'individualisation de milieux confinés favorables à la genèse d'argiles fibreuses, sous un climat chaud à alternance de périodes sèche et humide. E. (Algouti, 1991) A proposé une histoire paléogéographique pour le Sénonien du versant Nord du Haut-Atlas de Marrakech, depuis Jbel Sour à l'Est jusqu'à Imin'Tanout à l'Ouest, fondée sur l'étude des faciès, leur milieu de dépôt et leur évolution dans l'espace et dans le temps (Rhalmi, 1992)lors de son étude des étages Cénomanien et Turonien de la région d'Imini, a décrit les faciès sénoniens trouvés à Tighrmit-Est (Gara Assaoud) et à Ste Barbe et leur évolution diagénétique.(Boummane, 1995)a proposé un schéma d'évolution tectono-sédimentaire du bassin d'Erguita depuis le début du mésozoïque jusqu'au Mio-Pliocène. (Boutchich, 1997) a établi un découpage séquentiel, basé sur une étude sédimentologique et micropaléontologique, des séries sénoniennes du Bassin d'Essaouira.

\section{Contexte géographique}

La zone d'étude (Timstiguite) est située $23 \mathrm{~km}$ au Nord-est de la ville de Kelaat M'gouna, province de Tinghir région de Draa Tafilalet. Cette zone (Timstiguite) appartient à la bordure Sud de Haut Atlas centrale limité au Nord par l'accident Sub-atlasique, au Sud par le prolongement des formations de l'Anti Atlas oriental, à l'Est par le Haut Atlas orientale et à I'Ouest par le bassin d'Agadir (Fig.1).

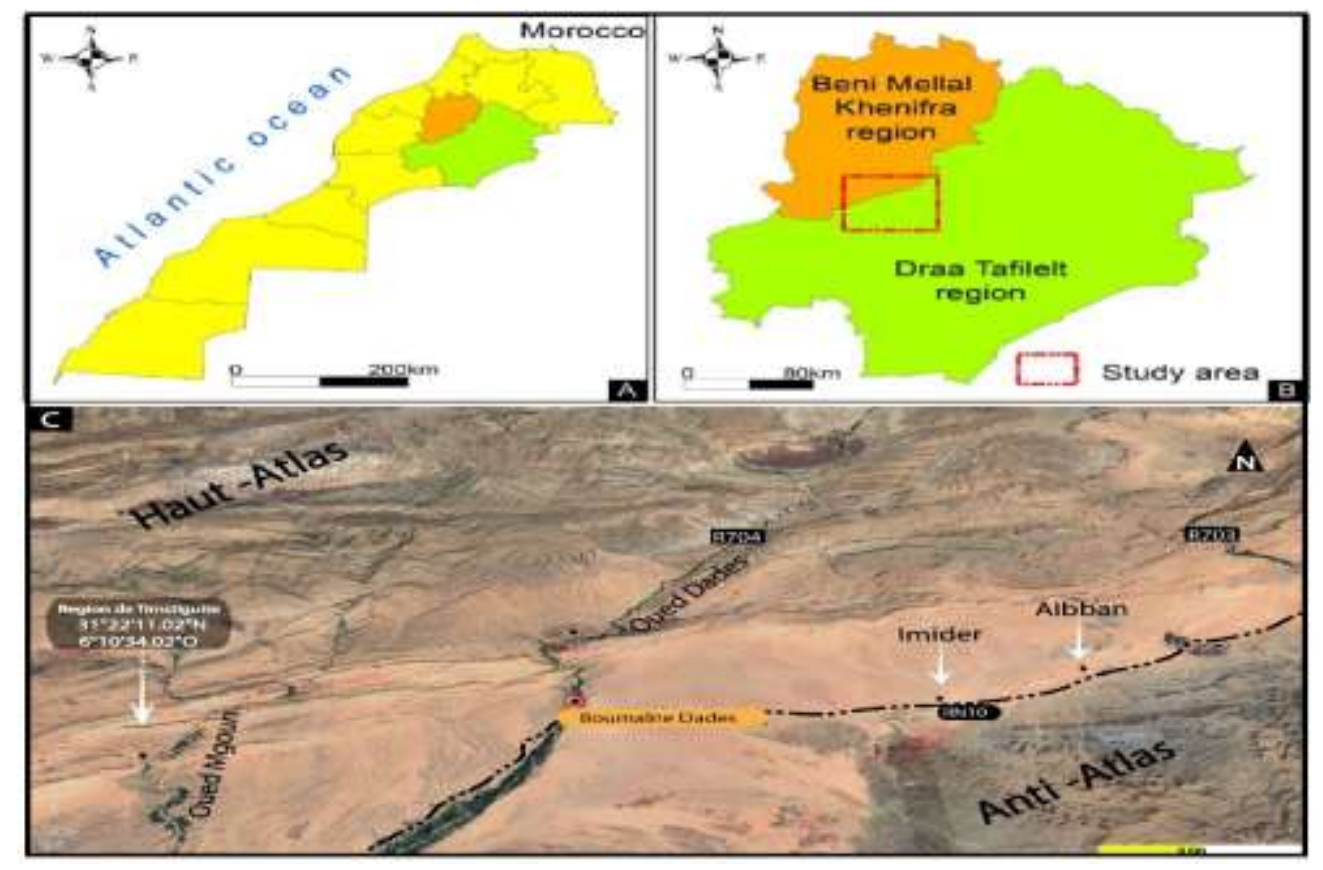

Figure.1 A : Carte du Maroc, B : Région de Draa Tafilalet. C ; Localisation géographique de la coupe géologique de référence réalisée (image google earth) 


\section{Contexte géologique}

La ville de Kelaat m'gouna appartient au bassin sédimentaire de Ouarzazate, Généralement ce bassin est caractérisé par l'affleurement du Néogène. Le sommet du M'Goun est constitué d'une longue crête qui dépasse ou approche les 4000 mètres d'altitude sur une longueur de dix kilomètres. En fait cette crête est un anticlinal. La roche, à la surface, est un calcaire dur. Son versant nord a été creusé par des glaciers lors des glaciations du Quaternaire si bien qu'aujourd'hui quatorze combes s'y alignent perpendiculairement à la ligne de faîte. Par contre la zone de Timstiguite, qui se trouve dans la partie sud de Haut Atlas Central est très connue par la présence du jurassique et crétacé en général et par le Sénonien en particulière dans cette zone étudiée. (Fig.2)

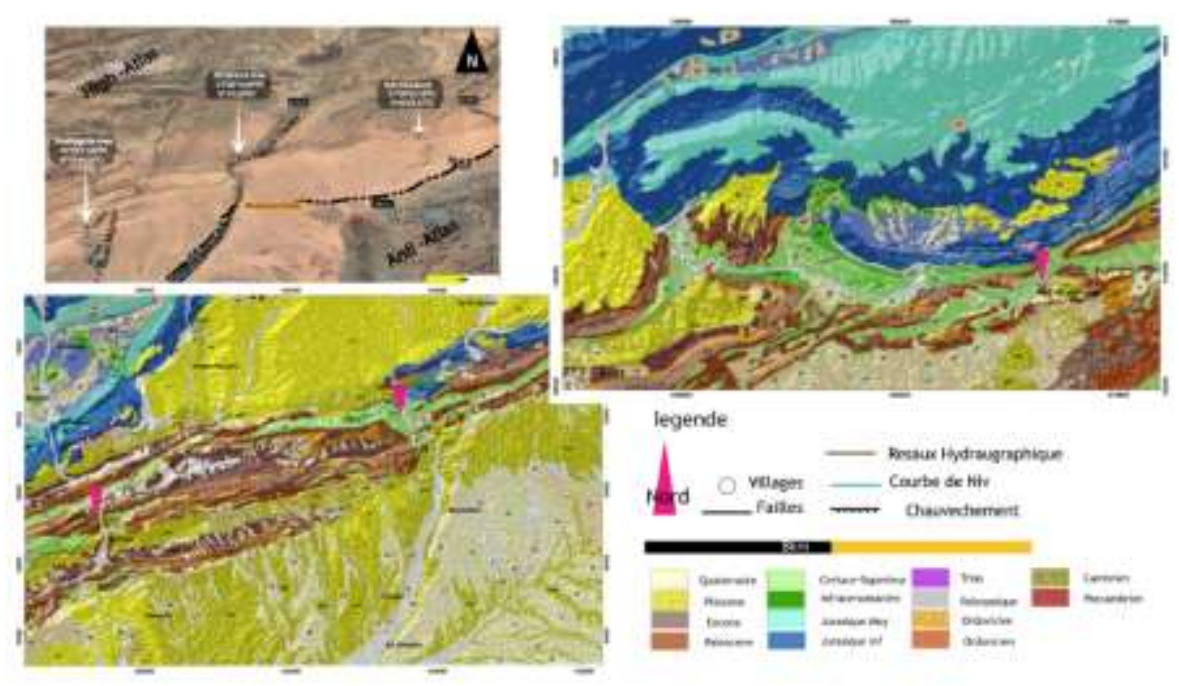

Figure.2. Extrait simplifiée des formations sénoniennes (voir légende) alignées ENE, sur base d'utilisation d'une carte géologique Jbel Saghrou Dades (1/200 000) et outils du système d'information géographique

\section{Contexte hydrologique}

L'Oued M'goun qui constitue le principal cours d'eau de la zone de Timstiguite, coule du Nord-est au Sud-ouest et verse dans I'Oued Dades, qui est le principale cours d'eau de la ville de Kelaat M'gouna. Alimentant en grande partie, le barrage El Mansour eddahbi, ce dernier fut construit en 1971 en aval de l'oued Draa. Il se situe à Ouarzazate à 90 km au Sud-ouest de Kelaat M'gouna, (Fig.3). Le réseau hydrographique de la zone de Dadès est formé par l'Oued Dadès avec son affluent M'goune, Le volume moyen annuel des ressources en eau de surface est estimé à $270 \mathrm{Mm} 3$ dont 147Mm3 apportés par Oued M'goun, $120 \mathrm{Mm} 3$ drainés par la partie amont d'oued Dadès. En ce qui concerne les eaux souterraines, le haut sous bassin d'Oued Dadès et le sous bassin d'oued M'goun sont constitués par les calcaires et dolomies du «Lias » et les grès du « Jurassique » qui sont largement fracturés et peuvent emmagasiner une part importante des eaux de pluies et de fonte de neige.

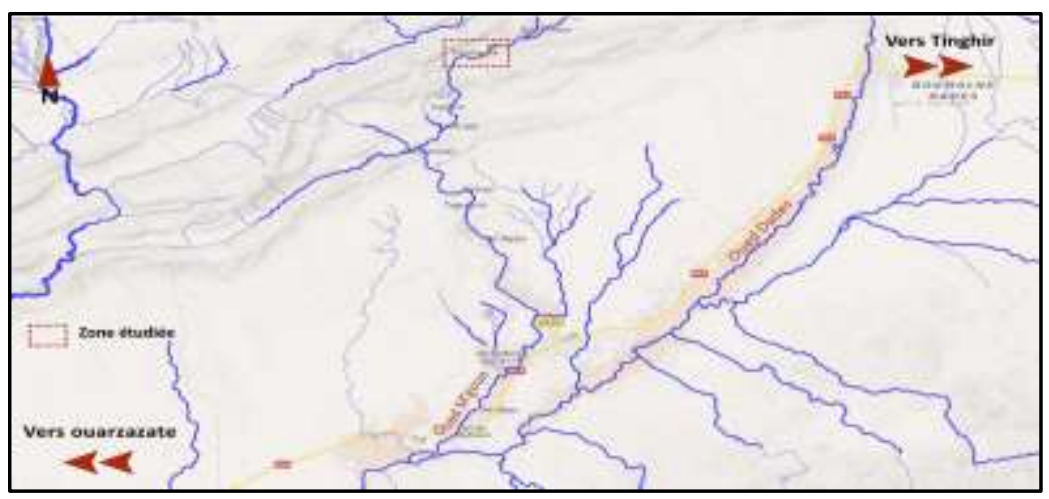

Figure.3 Réseau hydrographique de la zone étudiée

\section{Methodologie}


Le travail se concentre sur l'aspect stratigraphique et sédimentologique du crétacé supérieur (Sénonien) dans la region de Timstiguite, Les échantillons ont été prélevés, et les coordonnées notées (points GPS, dip...), les successions lithologiques ont été décrites de la base au sommet. L'objectif est généralement de répondre à ces questions : comment l'affleurement du Crétacé supérieur à Timstiguite se présente-t-il ? Quel est l'aspect dominant pour le faciès ? Comment les mesures tectoniques et les figures sédimentaires peuvent-elles nous aider à retracer les différentes paléoenvironnement ? Nous nous sommes également basés sur l'analyse des grains de quartz pour déterminer leur histoire paléogéographique, l'analyse de la composition de l'argile qui varie le long de la section réalisée, nous a beaucoup aidé à bien retracer certains milieux de dépôts des formations sénoniennes de Timstiguite

\section{Résultats et discussion}

\section{Localisation de la coupe de référence de la region d'étude (coupe Timstiguite)}

La coupe Timstiguite a été réalisée dans le petit village de Timstiguite avec une direction NW-SE. Elle est levée sur la rive gauche d'Oued M'goune, près de la route régionale menant à lghil M'goune. Les Cordonnées GPS : N 31 $21^{\prime} 57,0^{\prime \prime}$ W 6 10' 46,3"(Fig.4)

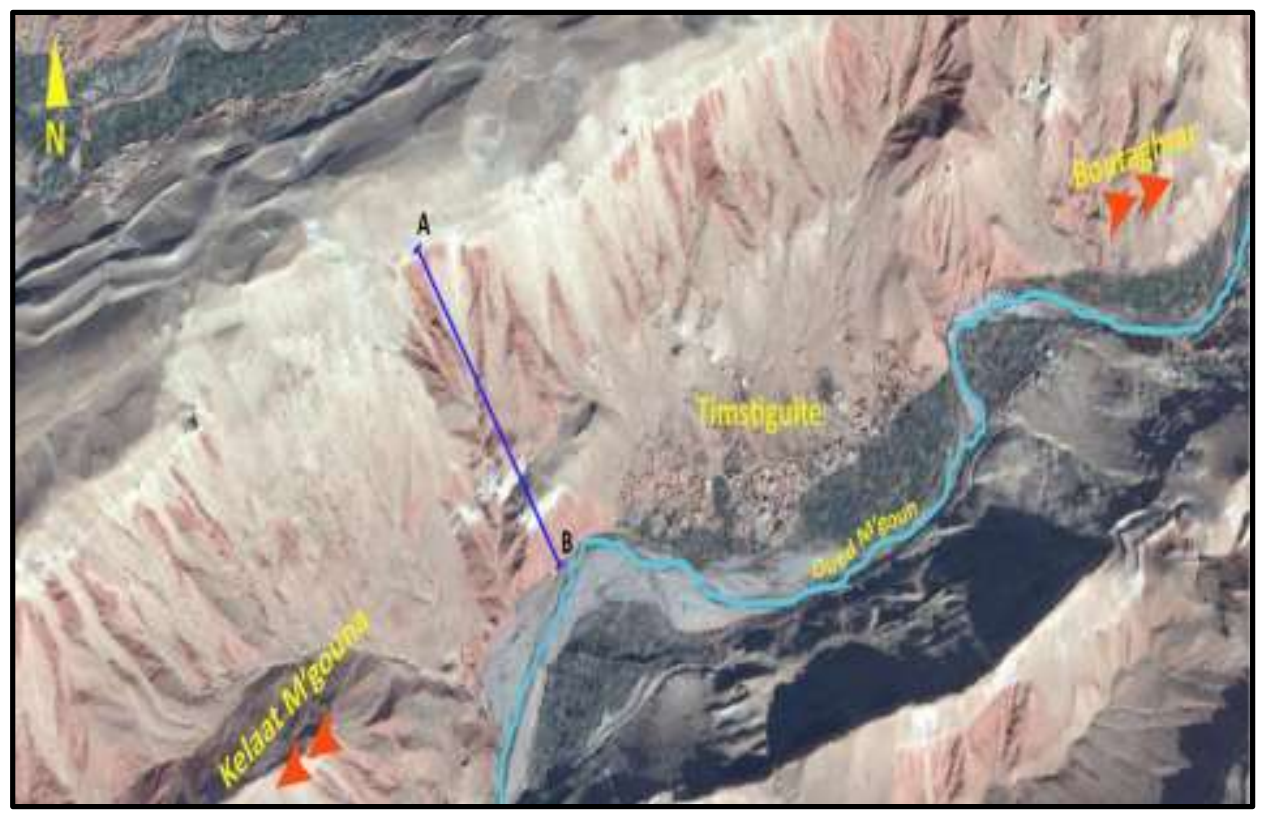

Figure.4 Situation géographique de la coupe réalisée (AB) "Timstiguite".

La coupe réalisée est caractérisée par une formation d'épaisseur d'environ de $500 \mathrm{~m}$. Elle est Composée d'un faciès détritique généralement rougeâtre. Elle s'organise d'une alternance de marne verte et d'argile rougeâtre à la base, et de grès fin avec des argiles très gypsifère au sommet. Cette formation sénonienne est limitée à la base par une barre de calcaire dolomitique jaunâtre (Cénomano-Turonien), et au sommet par une grande falaise de nature dolomitiques qui revient au Paléocène.

\section{Unité}

Pour rendre mieux l'interprétation et la compréhension de la coupe étudiée nous avons adopté un certain découpage de la coupe en trois unités bien distinguées.

Unité 1 : La première unité est délimitée à la base par une barre de calcaire dolomitique a surface oxydée, perforée et fossilisée qui traduit le Cénomano-Turonien (Fig.5.A). Après une succession de marne verdâtre et argile rouge avec la mise en place d'une barre carbonatée riche en lamellibranche, cette dernière est souvent déposée lors d'une première incursion marine qui a affecté tout le Sénonien de la région (Fig.5.B,C),vers le sommet de cette unité, nous constatons toujours les mêmes facies de marnes et argiles, mais parfois des faciès marno-argileux à gypse fibreux qui se répètent le long de cette unité 1(Fig.5.D) 

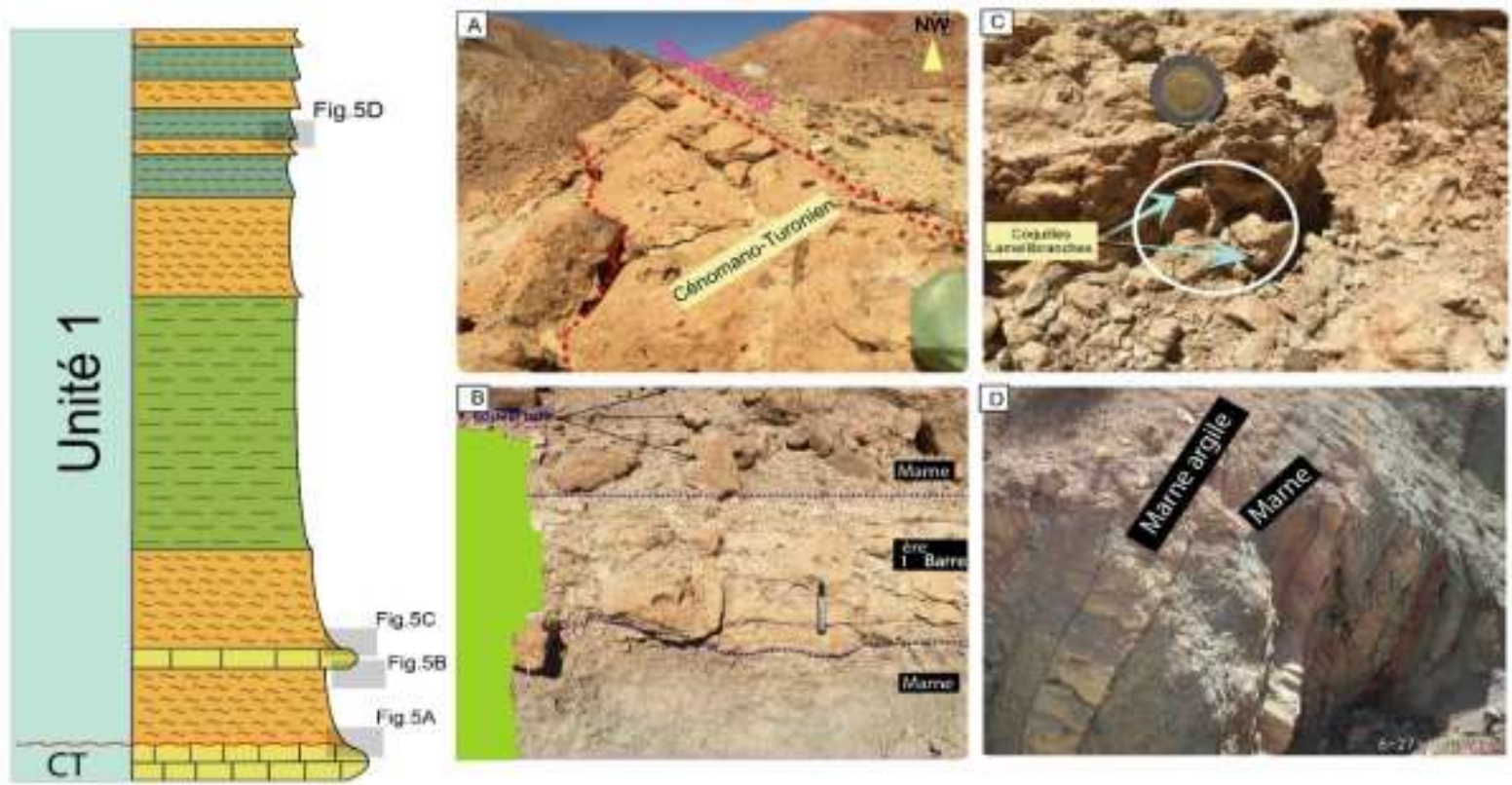

Figure .5 : à gauche : détail lithologique de l'unité 1 , à droite photos de formations lithologiques, certains figures et fossiles de même unité 1 ( $A$ : CenomanTuronien, $B: 1$ ère barre carbonatée, $C$ : Coquilles de Lamellibranches, $D$ : alternance marne et argile)

Unité 2 : Au début de cette deuxième unité, nous voyons une séquence de T0m d'épaisseur, présentant l'alternance des bancs de grès avec des intérbans d'argiles rouges meubles et parfois consolidés selon leurs conditions de dépôt, les grès montrent une variation de granulométrie de fine en grossière également nous distinguons entre trois type de grès, grès fin, un grès moyen et un grès grossier, ceci est toujours liée aux environnements de dépôts qui changent, Les gypses fibreux interstratifiés(Fig.6.C) dans les argiles et dans les marnes parfois occupent toute l'unité 2(Fig.6.A,B) .vers la partie supérieure de l'unité ,les couches de marne verte apparaissent, remplaçantes celles d'argiles, mais cette fois-ci elles sont très fossilisées (Fig.6.D),D'une manière sommaire nous pouvons annonce, que ces dépôts détritiques et évaporitiques reflètent des milieux lagunaires continentales.
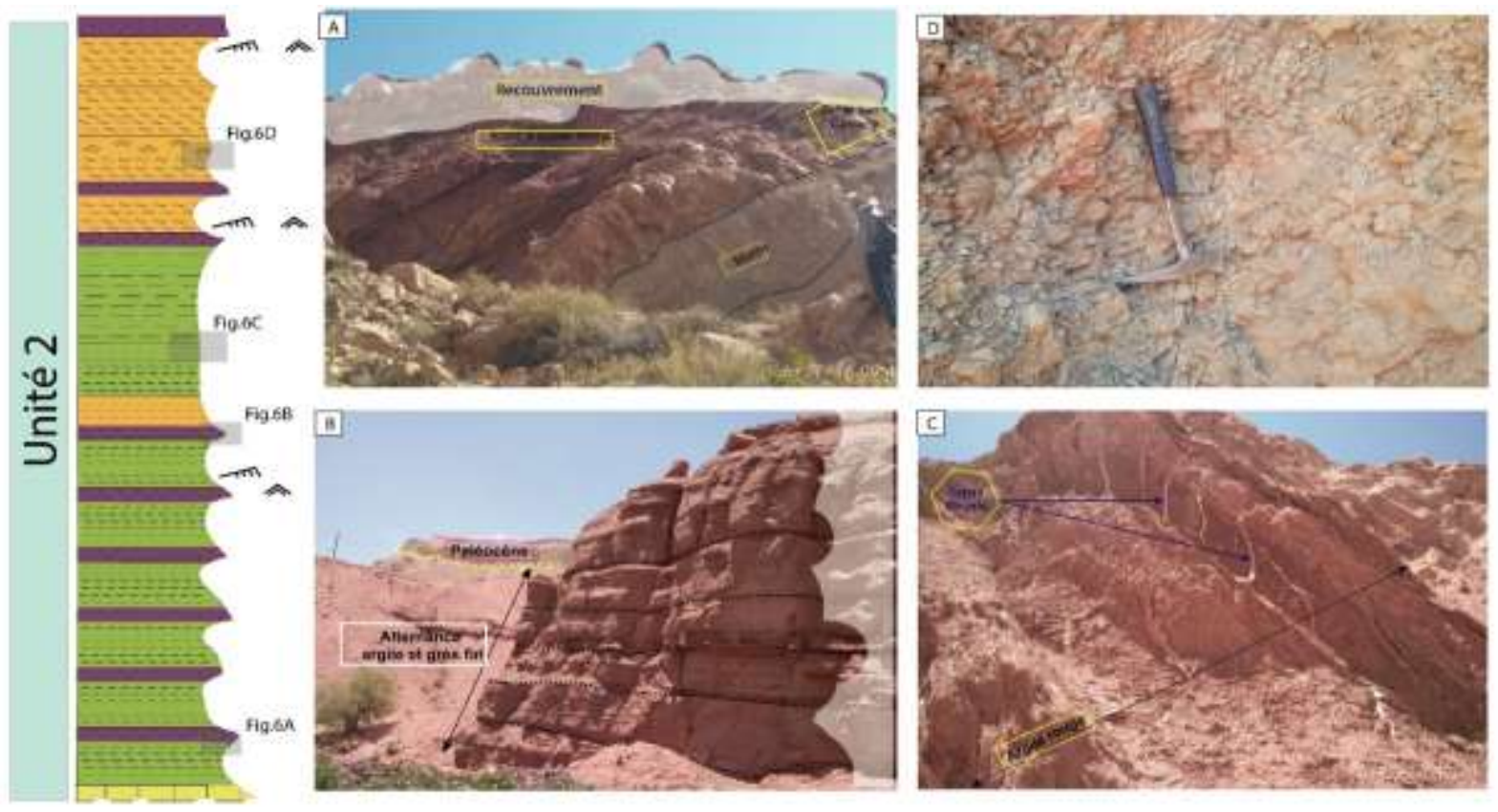

Figure .6 : à gauche : détail lithologique de l'unité 2 , à droite photos de formations lithologiques, certains figures et fossiles de même unité 21 ( $\mathrm{A}: 2$-ème barre carbonatée, B : alternance argile et grès fin, C: Gypse secondaire, D : Coquilles de Lamellibranches) 
Unité 3: L'unité 3 débute par une couche d'environ $2 \mathrm{~m}$ d'épaisseur de marne verdâtre. Ce faciès est surmonté par d'une couche de marne-argileuse, la grande partie lithologique affleurent dans cette troisième unité, Le long de toute l'unité nous remarquons la présence des gypse fibreux et dans certains endroits des gypses saccaroïde, Les niveaux gréseux au milieu de l'unité 3 présentent des bases érosives à granulométrie grossier indiquant un hydrodynamisme important, les figures sédimentaires sur les grès sont bien visible tel que la stratification parallèle et oblique, la présence des kerkoubs et autres indicateur de dépôt de facière détritique (Fig7 A, B et C) Cette unité et caractérisée au sommet par une couche de très fossilisée et parfois on trouve des gisements de fossiles de gastéropodes (Fig7. D). Finalement, toute la série du Sénonien se délimite par une barre de calcaire dolomitique de paléocène.
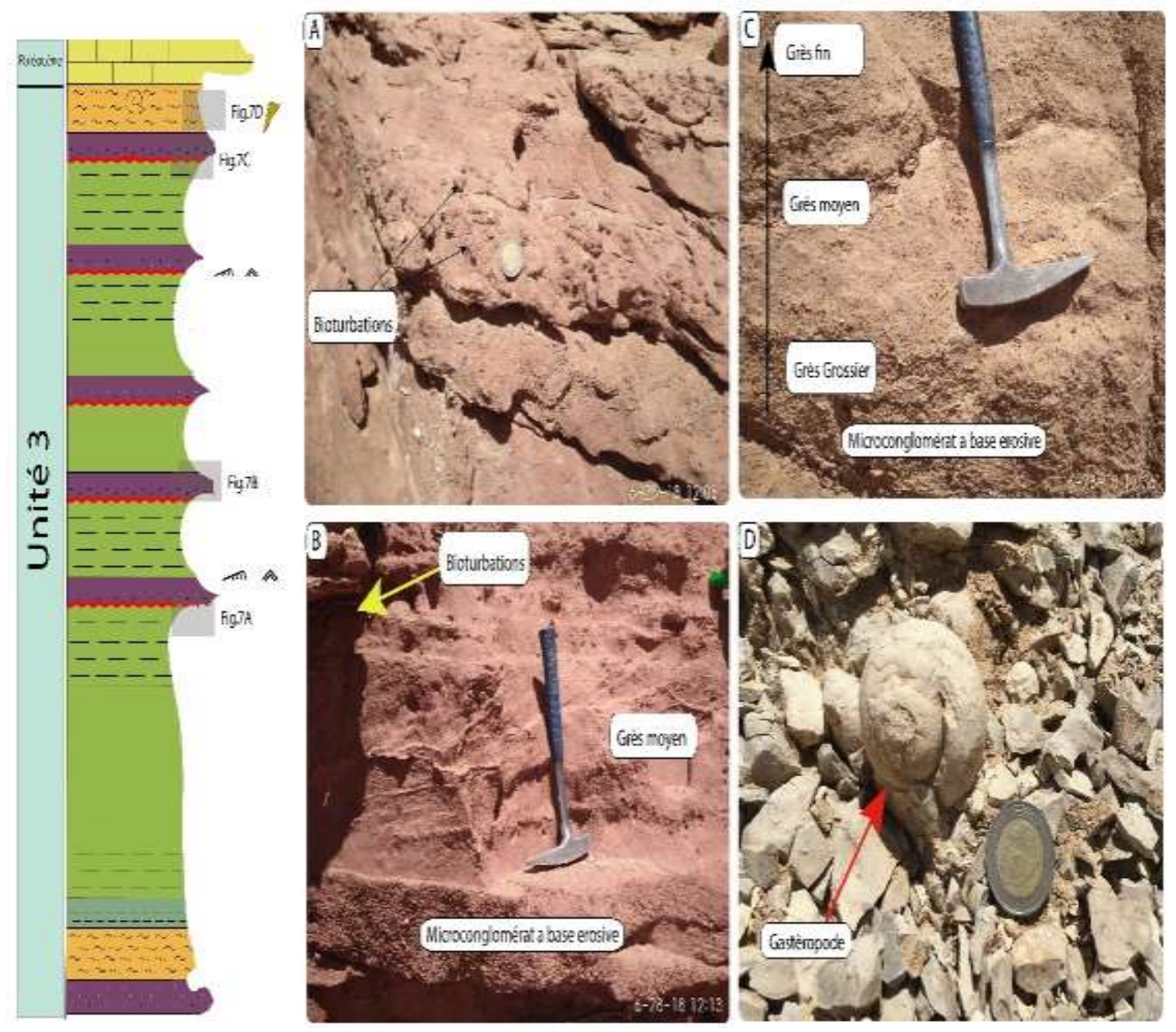

Figure .7 : à gauche : détail lithologique de l'unité 3, à droite photos de formations lithologiques, certains figures et fossiles de même unité 3 (A: Bioturbation, B : Base érosive, C : Granulométrie décroissante, D : marne a Gastéropode)

Le Sénonien de cette zone se considère comme une série d'un matériel rougeâtre, constitué généralement d'une alternance des argiles meubles et consolidées, et parfois des grès fins surtout dans la partie sommitale da la coupe mais aussi il y'avait des intercalations de marnes vertes partout, bien évidement le caractère gypsifère s'observe clairement dans la coupe réalisée, ce gypse est de nature fibreux secondaire. La série sénonienne entrecoupe par deux petites incursions marines, de nature calcaire jaunâtre, avec une épaisseur de deux mètres. Puis en milieu de la coupe, on note bien l'apparition de grès fin à couleur rougeâtre avec des lits de gypse fibreux. En ce qui concerne l'aspect fossilifère, nous avons marqués la présence des fossiles tel que Gastéropodes, lamellibranches et autres espèces. De point de vue tectonique, les dépôts sénoniens enregistrent des manifestations de la tectonique, généralement postérieure ; un réseau de failles qui a affecte toute la série (planche annexe). 


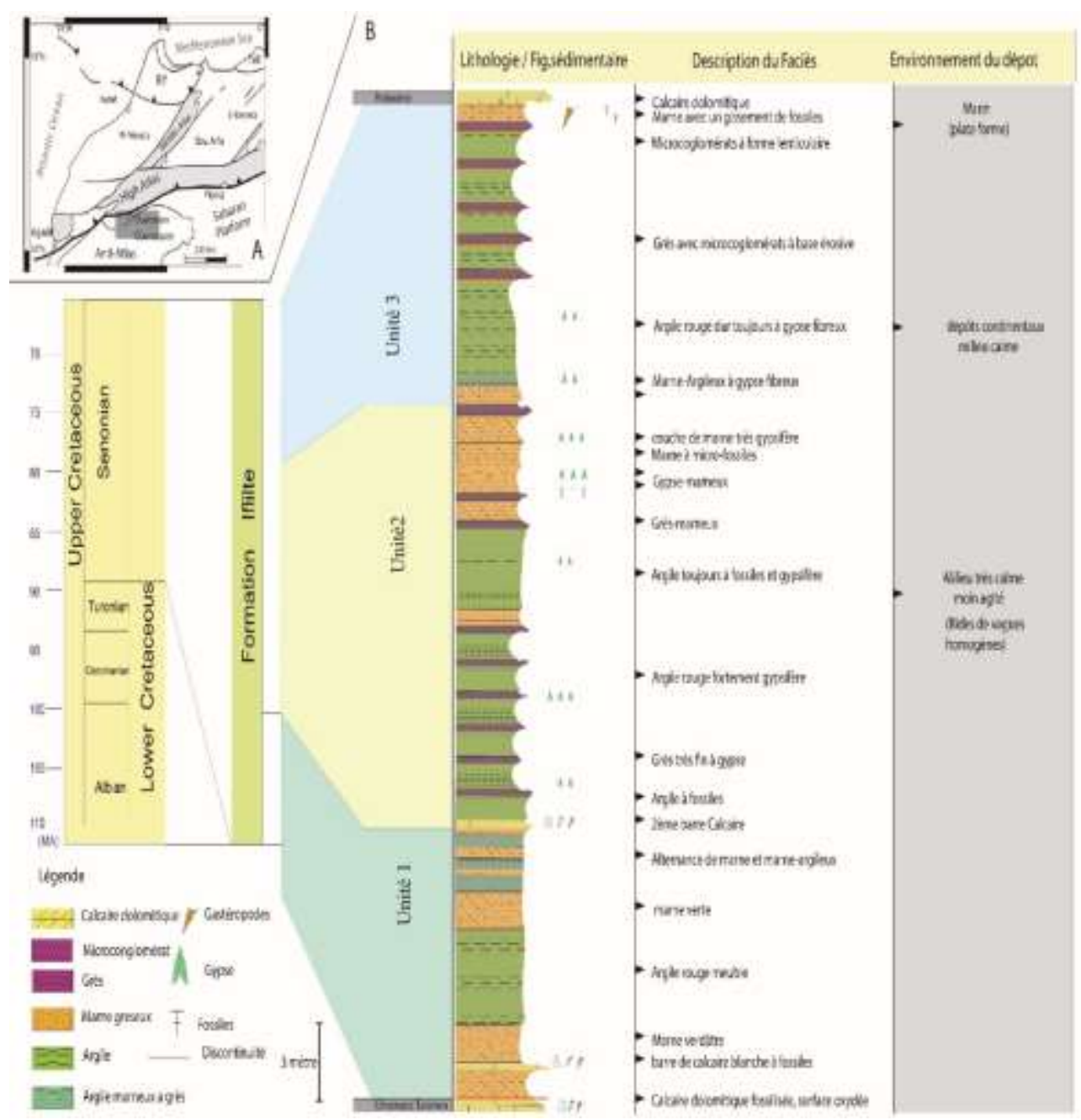

Fig.8 .A: Localisation de la coupe sur la bordure sud du Haut Atlas central Marocain B : Colonne litho stratigraphique de la coupe de Timstiguite avec certains détails de la lithologie et les figures sédimentaires et les milieux de dépôt

\section{Analyse exoscopique}

Le quartz est le minéral le plus abondant de la lithosphère, et en raison de sa dureté et sa résistance à l'abrasion et à la corrosion (absence de plan de clivage), il va représenter un grand pourcentage des sédiments terrigènes aussi bien continentaux que marins, essentiellement les sables. Le quartz est une espèce minérale du groupe des silicates, sous-groupe des tectosilicates, composé de dioxyde de silicium, ou silice, de formule chimique $\mathrm{SiO}$, avec des traces de différents éléments tels que $\mathrm{Al}, \mathrm{Li}, \mathrm{B}, \mathrm{Fe}, \mathrm{Mg}, \mathrm{Ca}$, $\mathrm{Ti}, \mathrm{Rb}$, $\mathrm{Na}, \mathrm{OH}$. Le quartz, provenant de la désagrégation des roches sous l'action de divers agents d'érosion (eau, glace et vent), va se retrouver le long des cours d'eau, aux bords des mers, ou dans les régions désertiques. En milieu fluvial, les grains s'usent en fonction de la durée du transport et de son énergie (les grains restent généralement anguleux pour un transport court). L'usure affecte surtout la surface des grains et au cours du temps, on a une modification de leur forme (apparition de phénomorphes). Les grains de sable usés deviennent émoussés et luisants (milieu aquatique de longue durée), (Tableau.1). Ces caractéristiques du quartz permettent de réaliser de nombreuses analyses sédimentologiques se rapportant à sa forme. Celles abordées dans ce travail sont la morphoscopie et l'exoscopie. 
Tableau. 1 Caractéristique du minéral du quartz

\begin{tabular}{|c|c|c|c|c|}
\hline Couleur & Clivage / Cassure & Dureté & Densité & Note \\
\hline $\begin{array}{l}\text { Incolore, blanc, gris, } \\
\text { jaune pâle, brun }\end{array}$ & $\begin{array}{c}\text { aucun clivage } \\
\text { cassure conchoïdale et } \\
\text { aussi cristalline }\end{array}$ & 7 & 2,65 & $\begin{array}{l}\text { Le quartz est le minéral le } \\
\text { plus commun du sable }\end{array}$ \\
\hline
\end{tabular}

L'étude exoscopique des grains de quartz montre d'une part l'existence de plusieurs types d'érosion mécanique et chimique, et d'autre part la présence de figures de dissolution sur la surface de plusieurs grains de quartz. Donc on peut constater que : Chaque grain à dépôts siliceux globuleux est le témoin d'un transport fluviatile de moyenne énergie, en raison de leur abondance et de l'absence de marques sur leur surface, ils n'ont subi qu'un très bref transport fluviatile de moyenne énergie. II s'agit des grains de quartz d'échantillons : (Fig.9.a, b et c) évoluent probablement dans un milieu confiné(M'RABET et al., 1978). Des grains montrent des arêtes plus émoussées : (Fig.9.b et c) et d'autres dévoilent des traces de chocs ainsi que des traces de dissolution en $V$ sur la surface des cassures a. Ces dernières peuvent correspondre à des traces de chocs et de frottements entre les grains en milieu aquatique de haute énergie (Flageollet, n.d.). Le début de polissage des cassures est le témoin d'un transport fluviatile de moyenne énergie, postérieur au transport précédent, il s'agit des grains de quartz d'échantillons (Fig.9.b et c) certaines cassures très nettes, fraîches dessinant des arêtes vives comme c'est le cas pour les échantillons a. Ces cassures se sont produit le long des niveaux de moindre résistance, et montrent que ces grains sont d'abord évolués en milieu fluviatile à haute énergie (Fig.9)

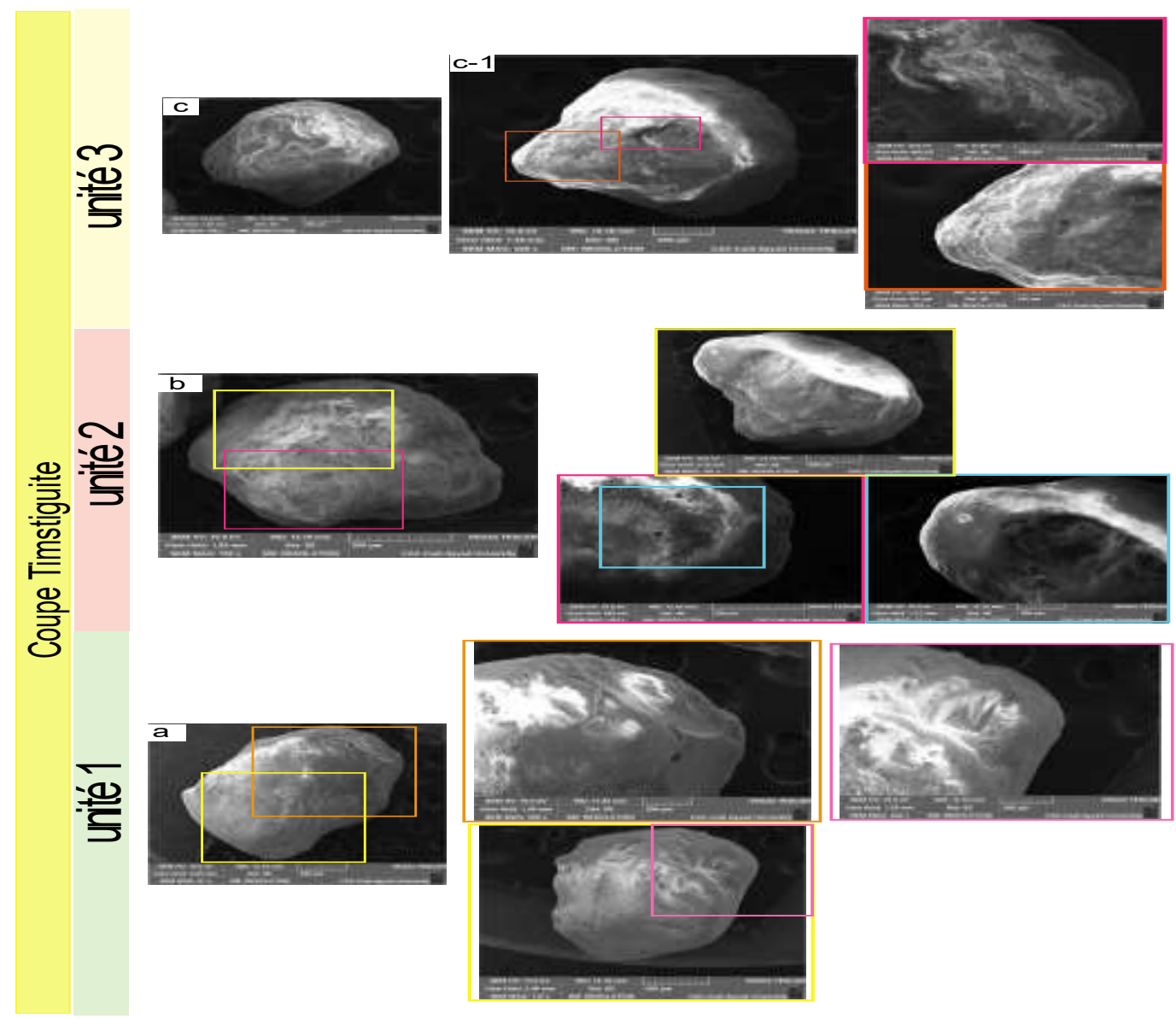

Figure.9 Analyse de la surface (exoscopie par MEB) des grains de quartz des échantillons a b et c des trois unités décrites 


\section{Cortège argileux}

Minéralogiquement, les argiles correspondent à des minéraux alumino-silicatés, le plus souvent de la famille des phyllosilicates: ce sont des particules à base d'aluminium et de silicium oxydés, arrangées en feuillets de très faible épaisseur (entre 7 et $14 \AA$ ). Compte tenu de la granulométrie des particules et de leur structure (association de feuillets de très faible épaisseur), les matériaux argileux développent des surfaces spécifiques très élevées, comprises entre 5 et $800 \mathrm{~m}^{2} / \mathrm{g}$, ce qui leur confère des propriétés d'adsorption très importantes(Caillère, 1982; Monjuvent.1973).Parmi ceux-ci, nous nous limiterons plus particulièrement à trois familles importantes pour notre étude : les muscovites, les illites et les smectites. Les cortèges argileux rencontrés au niveau de la coupe de de Timstiguite (Fig.10) montrent l'omniprésence de l'illite et la muscovite dans les trois unités, la présente de l'Illite suggère une active érosion de l'arrière-pays. Elle proviendrait vraisemblablement de l'altération superficielle des minéraux phylliteux préexistants. Son association avec la Muscovite dans la plupart du sénonien semble indiquer un apport fluviatile des régions de piémont (Sittler \& Millot, 1964). Le minéral muscovite (0-30\%) est présent dans presque toute la section de la base au sommet, ce qui suggère plusieurs origines pour sa présence, des néoformations ou des altérations des roches marines (Jurassique) de Haut Atlas comme sources d'éléments constituants de ce type de minéral. Toute cette variation est liée à une histoire d'une période relativement stable tectoniquement et un isolement du milieu sous un climat chaud et aride
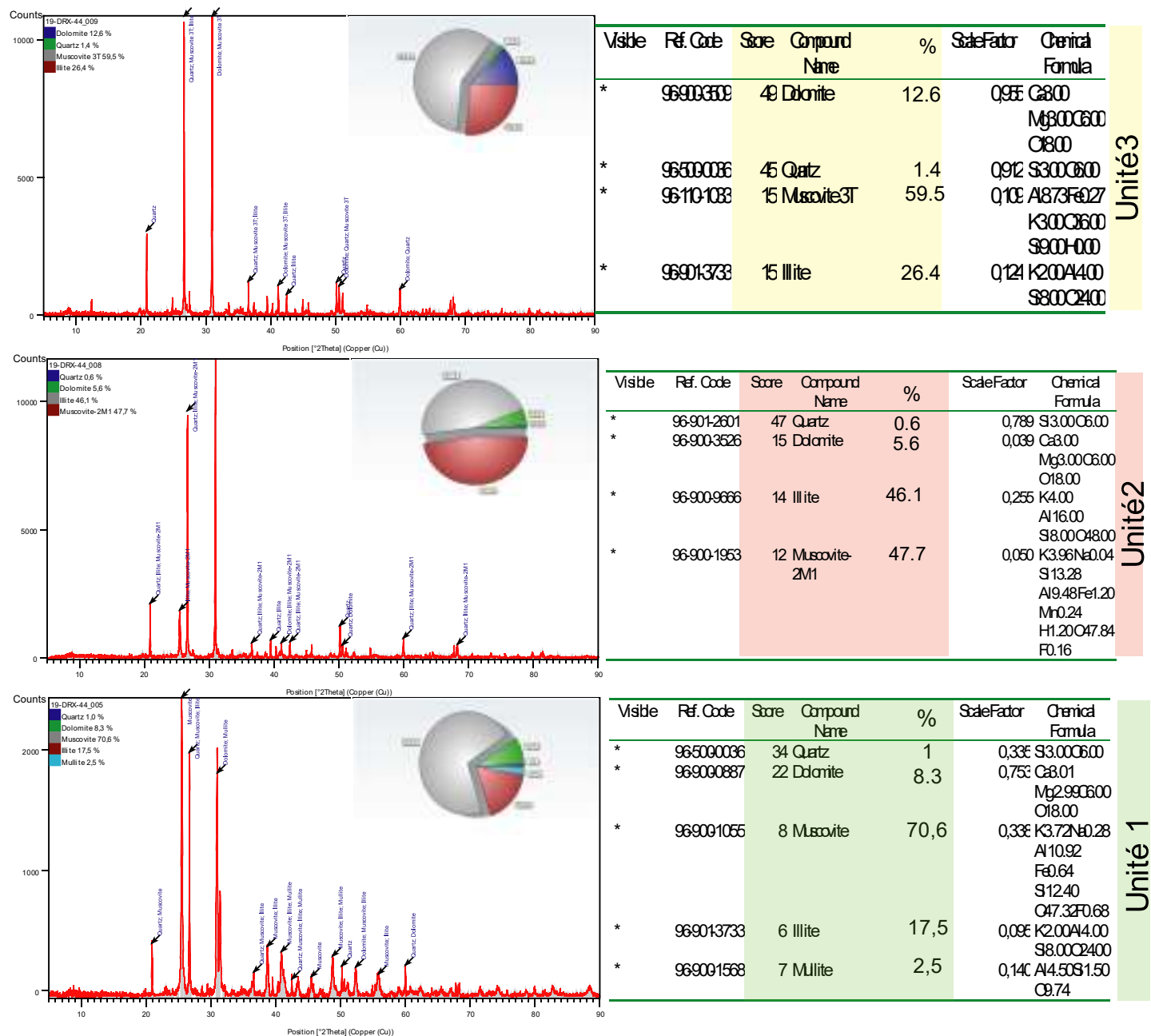

Figure.10 la variation de pourcentage de minéraux argileux le long de la coupe de Timtiguite (trois unités décrites)

\section{Paléogéographie}

La paléogéographie qu'on peut attribuer à ces terrains, ce sont des milieux continentales-lagunaires en se basant sur le côté lithologique de l'ensemble des formations géologiques qui constituent le sénonien de Timstiguite (Fig.8,11) 


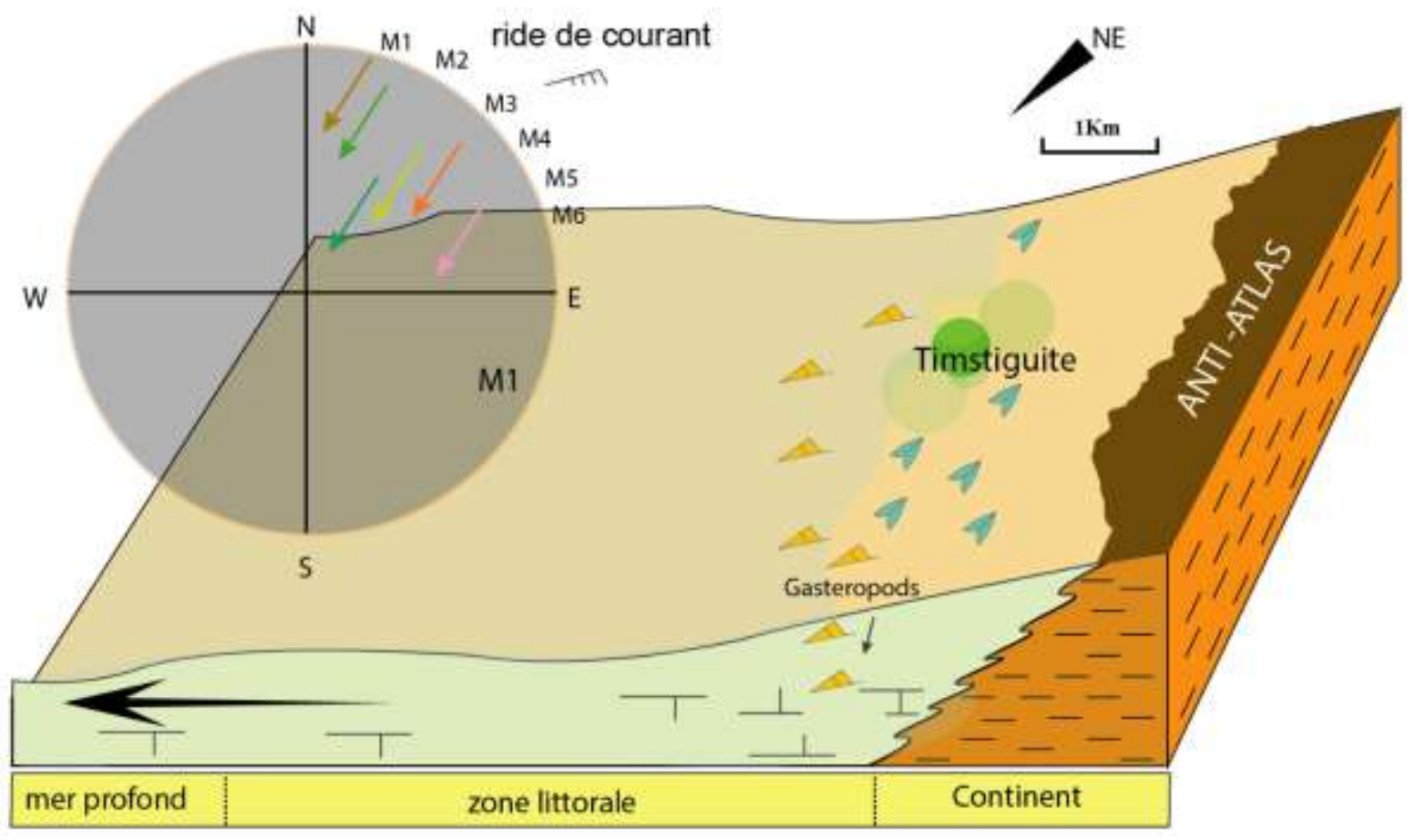

Figure.11. Modèle paléogéographique de la zone d'étude

\section{Conclusion}

Le Sénonien est un ensemble monotone de sables roses et rouges plus ou moins argileux, avec des lits de gypse secondaire. Ces formations lagunaires sont épaisses de $50 \mathrm{~m}$ à $100 \mathrm{~m}$ près du Jbel Saghro et de $250 \mathrm{~m}$ à $300 \mathrm{~m}$ dans le bassin d'Timstiguite. Du point de vue tectonique, les calcaires cénomanoturoniens ont été déposés pendant une période relativement calme, alors que le Sénonien a été une période d'activité tectonique qui a entrainé la reprise de la subsidence du bassin et le retour à une sédimentation détritique, liée au soulèvement d'ensemble de cette zone qui est déjà soumise à l'érosion.

\section{Remerciements}

Nos sincères remerciements vont au Département de Géologie et au Centre d'Analyse et de Caractérisation de la Faculté des Sciences Semlalia Marrakech, Université cadi Ayyad, (Maroc) pour avoir garanti toutes les analyses exoscopiques du quartz et pour l'analyse des argiles également. Les auteurs sont très reconnaissants aux évaluateurs anonymes pour leurs précieux commentaires et suggestions, ainsi qu'au rédacteur en chef de la revue pour son examen attentif de l'article qui a permis de présenter le travail de manière lucide.

\section{Références}

[1] Algouti, A. (1991). Turonien supérieur et Sénonien du versant nord du Haut-Atlas de Marrakech: Caractérisation sédimentologique et stratigraphique. https://toubkal.imist.ma/handle/123456789/1834.

[2] AZZAOUI, K. (1986). Le sénonien du Jbel Sour (versant nord du haut atlas de Marrakech).

[3] Behrens, M., Krumsiek, K., Meyer, D. E., Essen, A. S., Siehl, A., Stets, J., ... \& Wurster, P. (1978). Sedimentationsabläufe im Atlas-Golf (Kreide Küstenbecken Marokko). Geologische Rundschau, 67(2), 424-453.

[4] Boummane, K. (1995). Évolution tectonique et sédimentaire du bassin méso-Cénozoïque d'Erguita Bordures sur de la chaîne intracratonique du haut Atlas.(Massif ancien de Marrakech; Maroc).

[5] Boutchich, K. (1997). Le Senonien du Bassin d'Essaouira (Atlas atlantique, Maroc): stratigraphie, sédimentologie et micropaléontologie.

[6] Brives, A. (1919). Le Suessonien dans le Maroc central. Comptes Rendus de l'Académie des Sciences, 168, 776-777.

[7] Caillère, S. (1982). MINERALOGIE DES ARGILES. II: CLASSIFICATION ET NOMENCLATURE.

[8] Choubert, G., Du Dresnay, R., \& Hindermeyer, J. (1950). Sur les calcaires à Collenia de la région Safsaf-Aïn Chaïr. Notes Serv. géol. Maroc, 232, 997-999.

[9] Daoudi, L. (1991). Sédimentation et diagenèse des argiles du Jurassique-supérieur à l'Eocène dans le bassin du Haut-Atlas occidental (Maroc) (Doctoral dissertation, Lille 1).

[10] DUFFAUD, J., HEROS, R., GAUDILLIERE, R., \& CHOVIN, P. (1971, January). LE PROBLEME DE LA SURVEILLANCE DU NIVEAU DE POLLUTION D'UNE VILLE PAR LES GAZ D'ECHAPPEMENT DES VEHICULES AUTOMOBILES—CAS D'UN TUNNEL AUTOROUTIER: PROBLEM OF SURVEILLANCE OF THE LEVEL OF A TOWN'S POLLUTION FROM MOTOR CAR EXHAUST GASES. CASE OF A HIGHWAY TUNNEL. In PrOCeedingS 
of the Second International Clean Air Congress (pp. 16-22). Academic Press.

[11] FERRANDINI, J., \& LEMARREC, A. (1982). THE JURASSIC TO PALEOGENE COVER OF THE HIGH ATLAS OF MARRAKECH (MOROCCO) IS ALLOCHTONOUS IN THE ZONE-DES-CUVETTES OF AIT-OURIR. COMPTES RENDUS DE L ACADEMIE DES SCIENCES SERIE II, $295(9), 813-816$.

[12] Flageollet, J. C. (1981). Aspects morphoscopiques et exoscopiques des quartz dans quelques sols ferrallitiques de la région de Cechi (Côte d'Ivoire). Cahiers ORSTOM, Série Pédologique, 18, 111-121.

[13] Monjuvent, G., \& Uselle, J. (1973). Interpretation des «argiles» d'Eybens d'apres la sedimentologie du sondage INQUA 1969. Quaternaire, $10(2), 103-128$.

[14] Moret, L. (1925). Enquête critique sur les ressources minérales de la province de Savoie. In Annales Université de Grenoble (Vol. 3).

[15] M'RABET, A., M'RABET, A., \& RIBAULT, L. (1978). ANALYSE EXOSCOPIQUE DES SEQUENCES SABLEUSES DU BARREMIEN (FORMATION BOUDINAR) DE LA TUNISIE CENTRALE.

[16] Rhalmi, M. (1992). Les systèmes sédimentaires cénomano-turoniens et sénoniens de la région manganésifère d'Imini (Haut-Atlas central, Maroc) et leur évolution diagénétique (Doctoral dissertation, Dijon).

[17] Sittler, C., \& Millot, G. (1964). Les climats du Paléogène français reconstitués par les argiles néoformées et les microflores. Geologische Rundschau, 54(1), 333-343. C. Sittler and G. Millot, Les climats du paléogène franÇais reconstitués par les argiles néoformées et les microflores, Geol. Rundschau, 54(1), 333-343, May 1964, doi: 10.1007/BF0182118

[18] Stets, J., \& Wurster, P. (1982). Atlas and Atlantic-structural relations. In Geology of the Northwest African continental margin (pp. 69-85). Springer, Berlin, Heidelberg.

\section{Annexe}
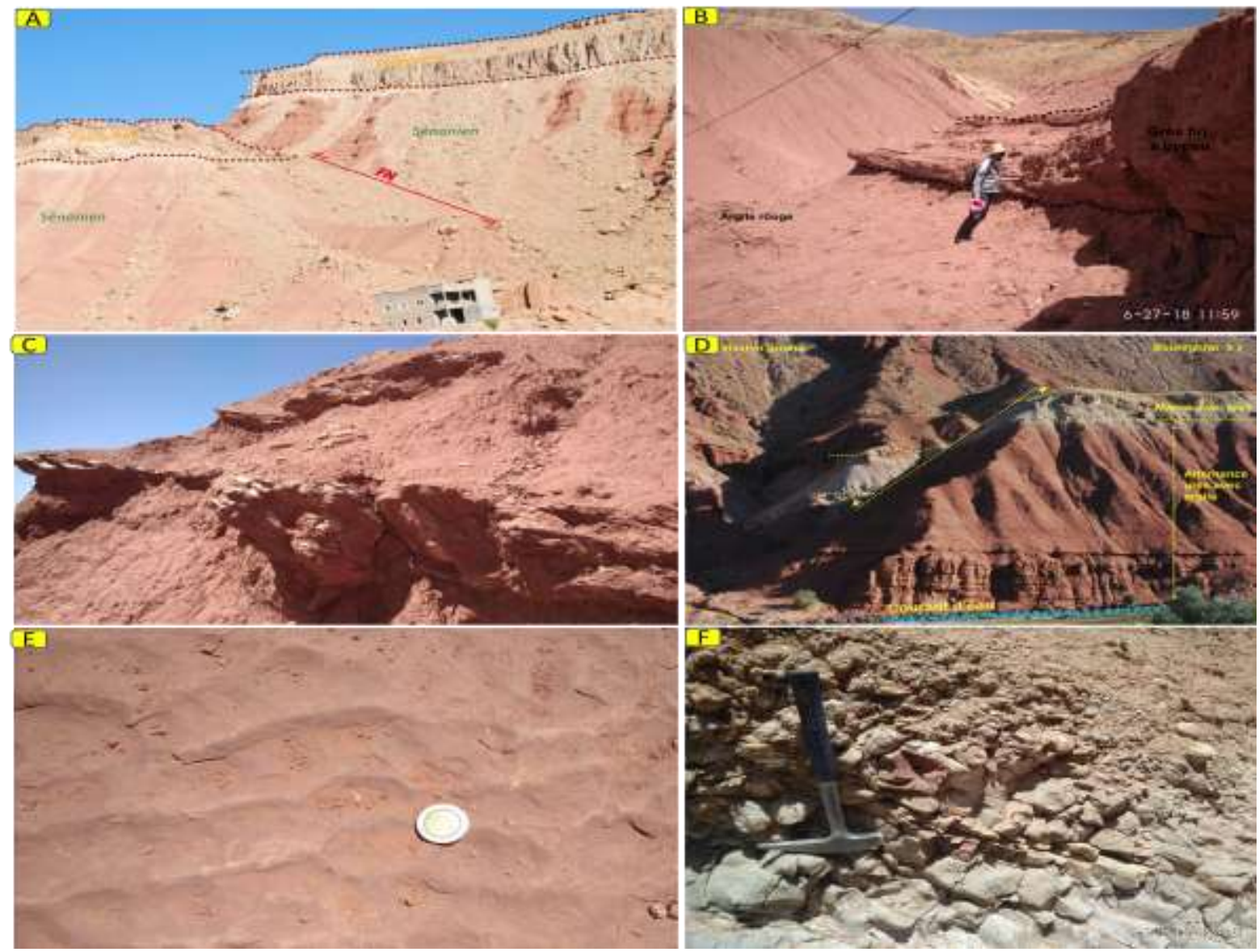

Planche 1. Photos prises lors de mission du terrain, A) basculement du paléocène, $B$ ) alternance grès argile de l'unité $2, C$ ) grès a gypse de l'unité 2, D) alternance marne argile de l'unité 1, E) Ride de lamination, E) Accumulation de gastéropodes de l'unité 3 